\title{
2-(2-[2-Dimethylaminothiazol-5-yl]Ethenyl)-6- (2-[Fluoro]Ethoxy)Benzoxazole: A Novel PET Agent for In Vivo Detection of Dense Amyloid Plaques in Alzheimer's Disease Patients
}

\author{
Yukitsuka Kudo $^{1}$, Nobuyuki Okamura ${ }^{2}$, Shozo Furumoto ${ }^{1}$, Manabu Tashiro ${ }^{3}$, Katsutoshi Furukawa ${ }^{4}$, \\ Masahiro Maruyama ${ }^{4}$, Masatoshi Itoh ${ }^{3}$, Ren Iwata ${ }^{5}$, Kazuhiko Yanai ${ }^{2}$, and Hiroyuki Arai ${ }^{4}$ \\ ${ }^{I}$ Tohoku University Biomedical Engineering Research Organization (TUBERO), Sendai, Japan; ${ }^{2}$ Department of Pharmacology, Tohoku \\ University School of Medicine, Sendai, Japan; ${ }^{3}$ Division of Cyclotron Nuclear Medicine, Cyclotron and Radioisotope Center, Tohoku \\ University, Sendai, Japan; ${ }^{4}$ Department of Geriatrics and Gerontology, Center for Asian Traditional Medicine, Tohoku University School \\ of Medicine, Sendai, Japan; and ${ }^{5}$ Division of Radiopharmaceutical Chemistry, Cyclotron and Radioisotope Center, Tohoku University, \\ Sendai, Japan
}

Extensive deposition of dense amyloid fibrils is a characteristic neuropathologic hallmark in Alzheimer's disease (AD). Noninvasive detection of these molecules is potentially useful for early and precise detection of patients with AD. This study reports a novel compound, 2-(2-[2-dimethylaminothiazol-5-yl]ethenyl)-6(2-[fluoro]ethoxy)benzoxazole (BF-227), for in vivo detection of dense amyloid deposits using PET. Methods: The binding affinity of BF-227 to amyloid- $\beta(A \beta)$ fibrils was calculated. The binding property of BF-227 to amyloid plaques was evaluated by neuropathologic staining of $A D$ brain sections. Brain uptake and in vivo binding of BF-227 to $A \beta$ deposits were also evaluated using mice. For clinical evaluation of ${ }^{11} \mathrm{C}-\mathrm{BF}-227$ as a PET probe, 11 normal (healthy) subjects and 10 patients with $A D$ participated in this study. Dynamic PET images were obtained for 60 min after administration of ${ }^{11} \mathrm{C}-\mathrm{BF}-227$. The regional standardized uptake value (SUV) and the ratio of regional to cerebellar SUV were calculated as an index of ${ }^{11} \mathrm{C}-\mathrm{BF}-227$ retention. The regional tracer distribution in AD patients was statistically compared with that of aged normal subjects on a voxel-by-voxel basis. Results: BF-227 displayed high binding affinity to synthetic $A \beta 1-42$ fibrils ( $\mathrm{K}_{\mathrm{i}}$ [inhibition constant], $\left.4.3 \pm 1.5 \mathrm{nM}\right)$. Neuropathologic staining has demonstrated preferential binding of this agent to dense amyloid deposits in AD brain. Moreover, a biodistribution study of this agent revealed excellent brain uptake and specific labeling of amyloid deposits in transgenic mice. The present clinical PET study using ${ }^{11} \mathrm{C}-\mathrm{BF}-227$ demonstrated the retention of this tracer in cerebral cortices of AD patients but not in those of normal subjects. All AD patients were clearly distinguishable from normal individuals using the temporal SUV ratio. Voxel-by-voxel analysis of PET images revealed that cortical BF-227 retention in $A D$ patients is distributed primarily to the posterior association area of the brain and corresponded well with the preferred site

Received Oct. 23, 2006; revision accepted Jan. 20, 2007.

For correspondence or reprints contact: Nobuyuki Okamura, MD, Department of Pharmacology, Tohoku University School of Medicine, 2-1, Seiryo-machi, Aoba-ku, Sendai 980-8575, Japan.

E-mail: oka@mail.tains.tohoku.ac.jp

COPYRIGHT @ 2007 by the Society of Nuclear Medicine, Inc. for neuritic plaque depositions containing dense $A \beta$ fibrils. Conclusion: These findings suggest that BF-227 is a promising PET probe for in vivo detection of dense amyloid deposits in $A D$ patients.

J Nucl Med 2007; 48:553-561

DOI: 10.2967/jnumed.106.037556

O ubstantial neuropathologic evidence suggests that the deposition of senile plaques (SPs) and neurofibrillary tangles (NFTs) represents the characteristic neuropathologic hallmark in Alzheimer's disease (AD) (1). Progressive accumulation of SPs is considered fundamental to the initial development of dementia. Extensive deposition of SPs in the brain is present even in very mild AD and precedes the presentation of cognitive impairment (2,3). Several antiamyloid drugs are under development for the treatment and prevention of AD (4). For early detection and preventive intervention for $\mathrm{AD}$, noninvasive imaging of neuropathologic lesions is a powerful strategy.

For this purpose, several imaging techniques have been developed that can noninvasively detect SPs in the brain using PET, SPECT, and MRI. Among these imaging modalities, PET is the most advanced and practical method for in vivo measurement of SP depositions. To achieve successful imaging using PET, various radiolabeled agents have been developed. Currently, 6OH-BTA-1 (PIB) is the most successful PET agent for in vivo amyloid imaging. This tracer sensitively detects amyloid fibrils in the brain and is proven to be useful for early diagnosis of AD (5-7).

However, amyloid- $\beta(\mathrm{A} \beta)$ deposition is also frequent in aging, even in cognitively intact individuals. Excessive identification of $\mathrm{A} \beta$ has a potential risk to misjudge the normal aging process with abnormal $A \beta$ deposition. In the 
normal aging process, noncompact or diffuse amyloid plaques containing less fibrillar $A \beta$ are deposited primarily in the brain. Brains from patients with $\mathrm{AD}$ are characterized by an anatomically widespread process of amyloid deposition and neuritic plaque formation containing dense amyloid fibrils (8). A shift of brain $A \beta$ from the soluble to the fibrillar form is closely associated with the onset of AD (9). Therefore, selective detection of dense amyloid fibrils would be advantageous to differentiate the normal aging process from $\mathrm{AD}$ with high specificity.

We have previously demonstrated a novel series of benzoxazole derivatives as promising candidates for an in vivo imaging probe of SPs (10-12). These derivatives showed comparatively high blood-brain barrier (BBB) permeability, high binding affinity for $A \beta$ aggregates, and high specificity for fibrillar $\mathrm{A} \beta$ deposits, suggesting potential merit for the early detection of AD-related pathologies. Herein we introduce an optimized derivative, 2-(2-[2-dimethylaminothiazol5-yl]ethenyl)-6-(2-[fluoro]ethoxy)benzoxazole (BF-227), as a PET probe for in vivo detection of dense amyloid deposits in humans.

\section{MATERIALS AND METHODS}

\section{Preparation of Compounds}

BF-227 (Fig. 1) and its $N$-desmethylated derivative (a precursor of $\left.{ }^{11} \mathrm{C}-\mathrm{BF}-227\right)$ were custom-synthesized by Tanabe R\&D Service Co. ${ }^{11} \mathrm{C}-\mathrm{BF}-227$ was synthesized from the precursor by $\mathrm{N}$-methylation in dimethyl sulfoxide (Fig. 1) using ${ }^{11} \mathrm{C}$-methyl triflate $(13,14)$. After quenching the reaction with $5 \%$ acetic acid in ethanol, ${ }^{11} \mathrm{C}-\mathrm{BF}-227$ was separated from the crude mixture by semipreparative reversedphase high-performance liquid chromatography and then isolated from the collected fraction by solid-phase extraction. The purified ${ }^{11} \mathrm{C}-\mathrm{BF}-227$ was solubilized in isotonic saline containing $1 \%$ polysobate- 80 and $5 \%$ ascorbic acid. The saline solution was filtersterilized with a $0.22-\mu \mathrm{m}$ Millipore filter for clinical use. The radiochemical yields were $>50 \%$ based on ${ }^{11} \mathrm{C}$-methyl triflate, and the specific radioactivities were $119-138 \mathrm{GBq} / \mu \mathrm{mol}$ at the end of synthesis. The radiochemical purities were $>95 \%$.

\section{In Vitro Binding Assays}

Binding affinities of the compounds for synthetic A $\beta 1-42$ aggregates were examined as described previously (10). Briefly, solid-form A $\mathrm{A} 1-42$ (Peptide Institute) was dissolved in $10 \mathrm{mM}$ potassium phosphate buffer ( $\mathrm{pH} 7.4)$ and incubated at $37^{\circ} \mathrm{C}$ for $40 \mathrm{~h}$. The binding assay was performed by mixing $100 \mu \mathrm{L}$ of aggregated
A $\beta 1-42$ with the appropriate concentration of ${ }^{125}$ I-labeled 2-(4methylamino)styryl-5-iodo-benzoxazole (BF-180) and 8\% ethanol. After incubation for $4 \mathrm{~h}$ at room temperature, the binding mixture was filtered and filters containing bound ${ }^{125}$ I ligand were counted using a $\gamma$-counter. The dissociation constant $\left(\mathrm{K}_{\mathrm{d}}\right)$ and maximum specific binding $\left(\mathrm{B}_{\max }\right)$ of BF-180 were determined. For inhibition studies, binding studies were performed using synthetic A $\beta 1-42$ aggregates. A mixture containing $50 \mu \mathrm{L}$ of BF-227, $50 \mu \mathrm{L}$ of 0.05 $n{ }^{125} \mathrm{I}-\mathrm{BF}-180,100 \mu \mathrm{L}$ of $100 \mathrm{nM} \mathrm{A} \beta 1-42$, and $800 \mu \mathrm{L}$ of $8 \%$ ethanol was incubated at room temperature for $4 \mathrm{~h}$. The mixture was then filtered through Whatman GF/B filters, and filters containing bound ${ }^{125} \mathrm{I}$ ligand were counted in a $\gamma$-counter. Values for the halfmaximal inhibitory concentration $\left(\mathrm{IC}_{50}\right)$ were determined from displacement curves of 3 independent experiments using Prism software (GraphPad), and values for the inhibition constant $\left(\mathrm{K}_{\mathrm{i}}\right)$ were determined using the Cheng-Prusoff equation.

\section{Measurement of Octanol/Water Partition Coefficients}

Phosphate-buffered saline (PBS) and 1-octanol (Wako) were saturated with 1-octanol and PBS, respectively, before use. BF227 was dissolved in 1-octanol and shaken with equal amounts of PBS for $30 \mathrm{~min}$ at room temperature. After centrifugation at 2,000 rpm for $15 \mathrm{~min}$, absorbency of the 1-octanol layer was measured at the peak wavelength of the absorbance spectrum of BF-227 using a Spectra Max 190 microplate reader (Molecular Devices). Octanol/water partition coefficients were determined by comparing absorbency with that before shaking with PBS. Each data point was performed in duplicate.

\section{BBB Permeability of BF-227 in Normal Mice}

Brain uptake of BF-227 was measured using ${ }^{11} \mathrm{C}$-labeled compound. The ${ }^{11} \mathrm{C}-\mathrm{BF}-227(1.1-6.3 \mathrm{MBq})$ was administered into the tail vein of male C57B6 mice ( $n=23$; mean weight, 28-32 g). Mice were then sacrificed by decapitation at 2, 10, 30, and $60 \mathrm{~min}$ after injection. The whole brain was removed and weighed, and radioactivity was counted using an automatic $\gamma$-counter. The percentage injected dose per gram of tissue $(\% \mathrm{ID} / \mathrm{g})$ was calculated by normalizing tissue counts to tissue weight. Each $\% \mathrm{ID} / \mathrm{g}$ value is expressed as a mean $\pm \mathrm{SD}$ of 3 or 4 separate experiments.

\section{Neuropathologic Staining}

Postmortem brain tissues from a 69-y-old man with autopsyconfirmed AD and an 81-y-old man with autopsy-confirmed physiologic aging were obtained from Fukushimura Hospital (Toyohashi, Japan). Experiments were performed under the regulations of the ethics committee of BF Research Institute. Serial sections $(6-\mu \mathrm{m}$ thick) from paraffin-embedded blocks of temporal cortex, striatum, and cerebellum were prepared in xylene and ethanol. Before
FIGURE 1. Chemical structures of BF227 and BF-168 and radiosynthesis of BF-227.

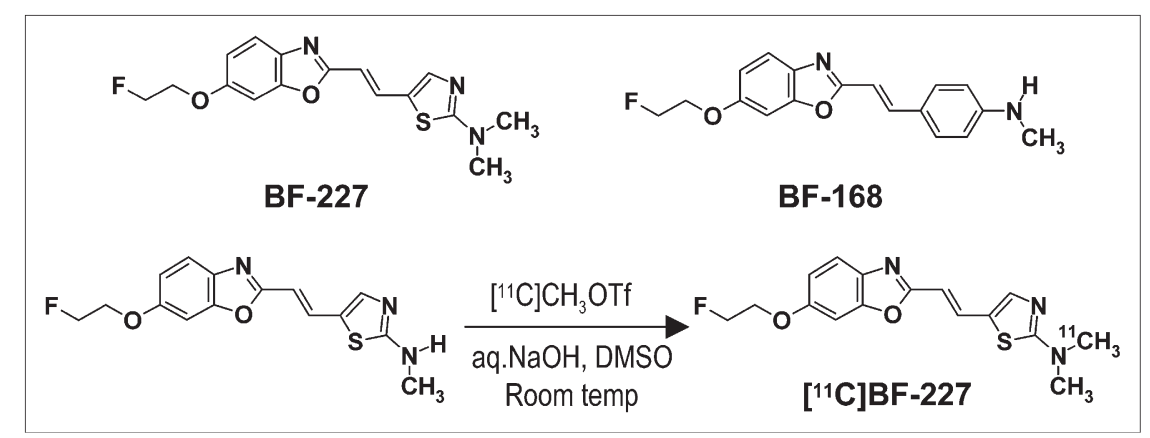


the staining of compounds, quenching of autofluorescence was performed as described previously. Quenched tissue sections were immersed in $100 \mu \mathrm{M}$ of BF-227 or $0.125 \%$ thioflavin-S solution containing $50 \%$ ethanol for 10 min. Sections stained with each compound were then dipped briefly into water and rinsed in PBS for $60 \mathrm{~min}$ before coverslipping with Fluor Save Reagent (Calbiochem); sections were examined using an Eclipse E800 microscope (Nikon) equipped with a V-2A filter set (excitation, 380-420 nm; dichroic mirror, $430 \mathrm{~nm}$; long-pass filter, $450 \mathrm{~nm}$ ). Sections stained with thioflavin-S were dipped briefly in tap water and in 50\% ethanol and then washed in PBS for $60 \mathrm{~min}$ before coverslipping; this was followed by fluorescent microscopy using a BV-2A filter set (excitation, 400-440 nm; dichroic mirror, 455 $\mathrm{nm}$; long-pass filter, $470 \mathrm{~nm}$ ). In addition, adjacent sections were immunostained using monoclonal antibody $(\mathrm{mAb})$ against $\mathrm{A} \beta$ (6F/3D; Dako A/S). After pretreatment with $90 \%$ formic acid for $5 \mathrm{~min}$, sections were immersed in blocking solution for $30 \mathrm{~min}$ and then incubated for $60 \mathrm{~min}$ at $37^{\circ} \mathrm{C}$ with $6 \mathrm{~F} / 3 \mathrm{D}$ at a dilution of 1:50. After incubation, sections were processed by the avidinbiotin method using a Pathostain ABC-POD(M) Kit (Wako) and diaminobenzidine tetrahydrochloride.

\section{Labeling of $\mathrm{A} \beta$ Deposits in Transgenic Mouse Brain}

Ex vivo plaque labeling with BF-227 was evaluated using PS1/ APPsw double transgenic mice $(n=2)$ and a wild-type mouse $(n=1)$ (male, 32-wk old) (15). A BF-227 solution containing $10 \%$ polyethylenglycol 400 and $0.1 \mathrm{~mol} / \mathrm{L} \mathrm{HCl}$ was administered into the tail vein at a dose of $4 \mathrm{mg} / \mathrm{kg}$. Mice were anesthetized using sodium pentobarbital $2 \mathrm{~h}$ after injection of BF-227; they were then perfused transcardially with ice-cold saline, which was followed by $4 \%$ paraformaldehyde in $0.1 \mathrm{M}$ PBS, and the brains were removed. After cryoprotection in 30\% sucrose/0.1 M PBS, $6-\mu \mathrm{m}$ frozen sections were cut using an OTF cryostat and imaged with no additional staining for fluorescent microscopy using a $\mathrm{V}-2 \mathrm{~A}$ filter set. The same sections were immunostained using $\mathrm{mAb}$ against $\mathrm{A} \beta(6 \mathrm{~F} / 3 \mathrm{D})$ as described earlier.

\section{Subjects and Patients in Clinical PET Study}

Eleven normal (healthy) control subjects, including 3 young normal subjects and 8 aged-matched normal subjects, and 10 probable $\mathrm{AD}$ patients underwent PET measurement of ${ }^{11} \mathrm{C}-\mathrm{BF}-227$ distribution in the brain (Table 1). AD patients were recruited through the Tohoku University Hospital Dementia Patients Registry. The diagnosis of $\mathrm{AD}$ was made according to the National Institute of Neurological and Communicative Diseases and Stroke/ Alzheimer's Disease and Related Disorders Association (NINCDSADRDA) criteria. The normal control group was recruited from volunteers, who were taking no centrally acting medication, had no cognitive impairment, and had no cerebrovascular lesion on MR images. No significant difference in age was apparent between the $\mathrm{AD}$ group and the aged normal control group. AD patients had significantly lower mean mini-mental status examination (MMSE) scores than normal control subjects. This study was approved by the ethics committee on clinical investigations of Tohoku University School of Medicine and was performed in accordance with the Declaration of Helsinki. After complete description of the study to the patients and subjects, written informed consent was obtained.

\section{Image Acquisition Protocols}

The protocol of the PET study was approved by the Committee on Clinical Investigation at The Tohoku University School of
TABLE 1

Subject Demographics

\begin{tabular}{|c|c|c|c|c|}
\hline Group & Subject & Sex & Age (y) & MMSE score \\
\hline \multirow{4}{*}{$\begin{array}{l}\text { Young normal } \\
\qquad(n=3)\end{array}$} & YN 1 & $\mathrm{M}$ & 36 & 30 \\
\hline & YN 2 & M & 37 & 30 \\
\hline & YN 3 & $M$ & 36 & 30 \\
\hline & Mean \pm SD & & $36.3 \pm 0.6$ & $30.0 \pm 0.0$ \\
\hline \multirow{9}{*}{$\begin{array}{l}\text { Aged normal } \\
\quad(n=8)\end{array}$} & AN 1 & M & 69 & 30 \\
\hline & AN 2 & $\mathrm{~F}$ & 70 & 29 \\
\hline & AN 3 & $\mathrm{~F}$ & 64 & 30 \\
\hline & AN 4 & $\mathrm{~F}$ & 65 & 30 \\
\hline & AN 5 & M & 67 & 30 \\
\hline & AN 6 & M & 69 & 30 \\
\hline & AN 7 & $M$ & 71 & 30 \\
\hline & AN 8 & $M$ & 59 & 30 \\
\hline & Mean $\pm S D$ & & $66.8 \pm 4.0$ & $29.9 \pm 0.4$ \\
\hline $\begin{array}{l}\text { All normal } \\
\quad(n=11)\end{array}$ & Mean $\pm S D$ & & $58.5 \pm 14.6$ & $29.9 \pm 0.3$ \\
\hline \multirow[t]{11}{*}{$\mathrm{AD}(n=10)$} & AD 1 & $\mathrm{~F}$ & 65 & 24 \\
\hline & AD 2 & $M$ & 75 & 19 \\
\hline & AD 3 & $\mathrm{~F}$ & 72 & 21 \\
\hline & AD 4 & $\mathrm{~F}$ & 82 & 18 \\
\hline & AD 5 & $\mathrm{~F}$ & 62 & 20 \\
\hline & AD 6 & $\mathrm{~F}$ & 68 & 21 \\
\hline & AD 7 & $M$ & 70 & 23 \\
\hline & AD 8 & $\mathrm{~F}$ & 85 & 23 \\
\hline & AD 9 & M & 78 & 14 \\
\hline & $A D 10$ & $\mathrm{~F}$ & 75 & 26 \\
\hline & Mean $\pm S D$ & & $73.2 \pm 7.3^{*}$ & $20.9 \pm 3.4^{* \dagger}$ \\
\hline $\begin{array}{l}{ }^{\star} P<0.05 \text { vs. } \\
{ }^{\dagger} P<0.05 \text { vs. } \\
\text { MMSE }=\text { mini- }\end{array}$ & $\begin{array}{l}\text { young normal } \\
\text { aged normal g } \\
\text {-mental state }\end{array}$ & $\begin{array}{l}\text { group } \\
\text { group } \\
\text { exami }\end{array}$ & ination. & \\
\hline
\end{tabular}

Medicine and the Advisory Committee on Radioactive Substances at Tohoku University. The ${ }^{11} \mathrm{C}-\mathrm{BF}-227$ PET study was performed using a SET-2400W PET scanner (Shimadzu). After intravenous injection of 211-366 MBq of ${ }^{11} \mathrm{C}-\mathrm{BF}-227$, dynamic PET images were obtained for 60 min $(23$ sequential scans: 5 scans $\times 30 \mathrm{~s}$, 5 scans $\times 60 \mathrm{~s}, 5$ scans $\times 150 \mathrm{~s}$, and 8 scans $\times 300 \mathrm{~s})$ with each subject's eyes closed. The T1-weighted MR images were obtained using a SIGNA 1.5-T machine (GE Healthcare).

\section{Image Analysis}

First, standardized uptake value (SUV) images of ${ }^{11} \mathrm{C}-\mathrm{BF}-227$ were obtained by normalizing tissue radioactivity concentration by injected dose and body weight. Subsequently, individual MR images were anatomically coregistered into individual PET images using Statistical Parametric Mapping software (SPM2; Welcome Department, U.K.) (16). Regions of interest (ROIs) were placed on individual axial MR images in the cerebellar hemisphere, striatum, thalamus, frontal cortex (Brodmann's areas [BA] $8,9,10,44,45,46$, and 47), lateral temporal cortex (BA 21, 22, 37, and 38), parietal cortex (BA 39 and 40), temporooccipital cortex (BA 18 and 19), occipital cortex (BA 17), medial temporal cortex (BA 27, 28, 34, and 35), pons, and subcortical white matter, as described previously (17). The ROI information was then copied onto dynamic PET SUV images, and regional SUVs were sampled using Dr.View/LINUX software (Asahi-Kasei Joho System). 
The interrater reliability for the ROI measurement was tested between 2 raters in 14 subjects and patients. The intraclass correlation coefficient was 0.95 in the frontal cortex and cerebellum, 0.97 in the lateral temporal and parietal cortices, and 0.98 in the medial temporal cortex. The correlation coefficient between these 2 measurements was 0.96 in the frontal cortex, 0.97 in the lateral temporal cortex, and 0.99 in the parietal cortex, medial temporal cortex, and cerebellum. SUVs between 40 and 60 min were averaged to calculate the SUVs for group comparison.

\section{Statistical Analysis}

For statistical comparison in the 3 groups, we applied the Kruskal-Wallis test, which was followed by Dunn's multiple comparison test. The difference in time-activity curves in ${ }^{11} \mathrm{C}$ BF-227 PET was also evaluated by repeated measures ANOVA, which was followed by the Bonferroni-Dunn post hoc test. For statistical comparisons of PET measurements in aged normal and $\mathrm{AD}$ groups, we used the Mann-Whitney $U$ test. Effect-size coefficients (Cohen's $d$ ) were also calculated for the evaluation of group differences in PET measurements. Statistical significance for each analysis was defined as $P<0.05$. Statistical comparison between images from normal control subjects and AD patients was performed on a voxel-by-voxel basis using SPM2 software (16). SUV summation images 30-60 min after injection were stereotactically normalized using individual MR images into a standard space of Talairach and Tournoux. The normalized images were smoothed using a $16 \times 16 \times 16 \mathrm{~mm}$ gaussian filter. The count of each voxel was normalized to the cerebellar ROI value, because cerebellum is reported to be a region free of fibrillar amyloid plaques in $\mathrm{AD}$ brain. Images of patients with $\mathrm{AD}(n=10)$ were compared with those of aged normal control subjects $(n=8)$ for between-group analysis $(P<0.001$, uncorrected; extent threshold, $k=200$ ). For the group analysis, a 2-sample $t$ test was used to detect differences between the AD and normal control groups.

\section{RESULTS}

\section{In Vitro Binding Study for $\mathbf{A} \boldsymbol{\beta}$ Fibrils}

In vitro binding assay indicated that BF-227 shows high binding affinity for $A \beta 1-42$ fibrils. $K_{i}$ for $A \beta 1-42$ fibrils in competitive binding assay using ${ }^{125} \mathrm{I}-\mathrm{BF}-180$ was $4.3 \pm 1.5$ $\mathrm{nM}$ in BF-227, comparable to levels previously reported for compound BF-168.

\section{Neuropathologic Staining in AD Brain Sections}

Neuropathologic examination using BF-227 indicated that amyloid plaques were selectively stained with BF-227 in AD brain sections (Fig. 2A). Especially, cored plaques were brightly stained with $\mathrm{BF}-227$, indicating that this compound preferentially binds to mature amyloid plaque. This staining pattern correlated well with $\mathrm{A} \beta$ immunostaining in adjacent sections (Fig. 2B, arrows). BF-227 staining was further compared with staining using thioflavin-S. In contrast to clear staining of SPs and NFTs with thioflavin-S (Fig. 2C), BF-227 primarily stained SPs, with faint staining of NFTs (Fig. 2B, arrowheads). No apparent staining was also observed in the temporal brain section of the aged normal case (Fig. 2D).

\section{BBB Permeability and Clearance from Normal Brain}

Next, we investigated whether BF-227 entered the brain in amounts sufficient for use as a PET agent. The $\log P$ value of BF-227 was 1.75 , close to that of BF-168 $(\log P=$ 1.79). Intravenous administration of BF-227 into normal mice indicated that this compound readily penetrated the BBB. Brain uptakes at 2, 10, 30, and $60 \mathrm{~min}$ after intravenous injection of ${ }^{11} \mathrm{C}-\mathrm{BF}-227$ were $7.9 \pm 1.3,3.7 \pm 0.37$, $1.4 \pm 0.36$, and $0.64 \pm 0.15 \% \mathrm{ID} / \mathrm{g}$, respectively. ${ }^{11} \mathrm{C}-\mathrm{BF}-$ 227 displayed double the initial uptake and faster washout

FIGURE 2. Neuropathologic staining of human brain sections by BF-227. Amyloid plaques are clearly stained with BF227 in $A D$ temporal brain sections (A). BF-227 staining correlates well with $A \beta$ immunostaining in adjacent sections $(B$, arrows). BF-227 faintly stains NFTs, in contrast to clear staining with thioflavin-S (C, arrowheads). In aged normal temporal cortex (D), no staining by BF-227 is observed. Bar in $\mathrm{A}-\mathrm{C}=50 \mu \mathrm{m}$; bar in $\mathrm{D}=200 \mu \mathrm{m}$.
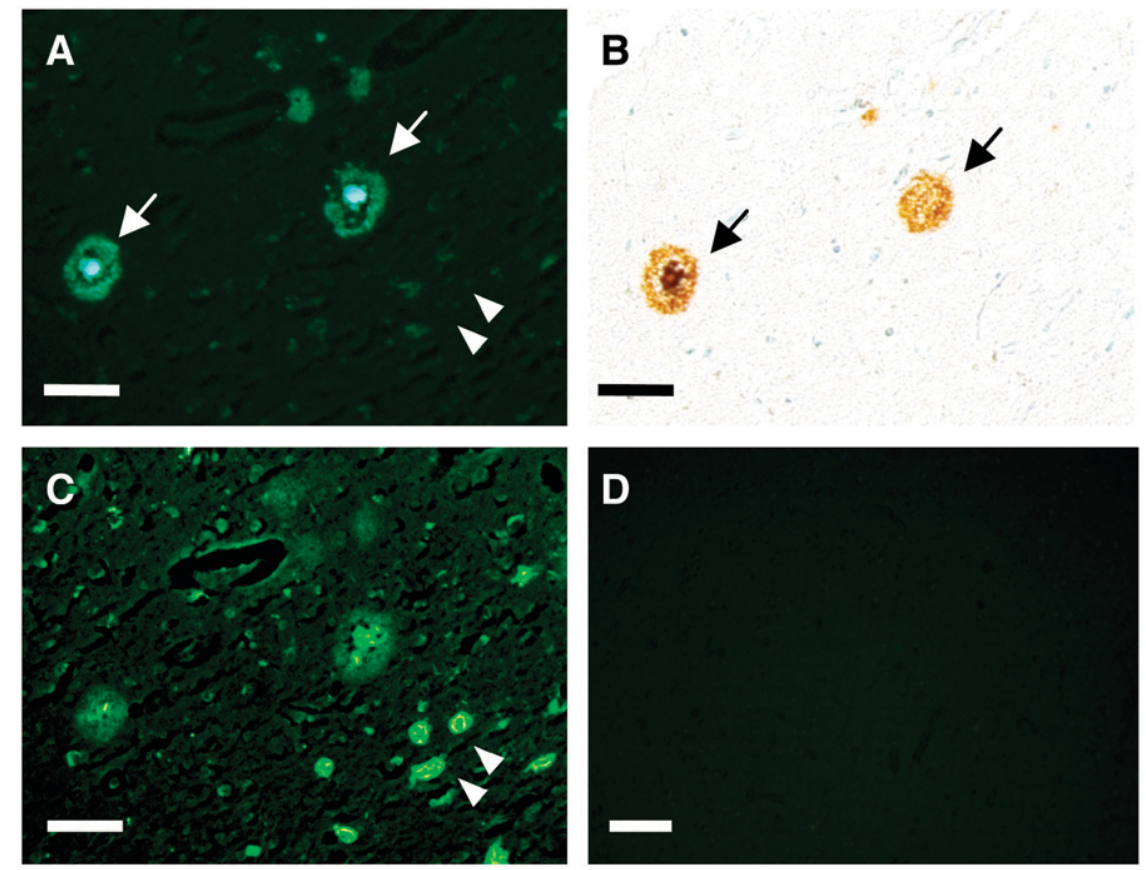
in normal brain tissue compared with those of ${ }^{18} \mathrm{~F}-\mathrm{BF}-168$ (3.9\% ID/g at $2 \mathrm{~min}$ after injection; $1.3 \% \mathrm{ID} / \mathrm{g}$ at $60 \mathrm{~min}$ after injection).

\section{Intravenous Administration of BF-227 in Transgenic Mice}

In vivo binding of nonlabeled $\mathrm{BF}-227$ to $\mathrm{A} \beta$ deposits was examined using PS1/APPsw double transgenic mice. After intravenous injection of $4 \mathrm{mg} / \mathrm{kg} \mathrm{BF}-227$, ex vivo observation of transgenic mouse brain slices showed numerous fluorescent spots in the neocortex and hippocampus (Figs. $3 \mathrm{~A}$ and $3 \mathrm{~B})$. In contrast, no fluorescent spots were detected in the wild-type mouse brain (Fig. 3C). Brain sections of transgenic mice were subsequently immunostained using $\mathrm{A} \beta$-specific antibody, and the distribution of plaques labeled with BF-227 corresponded well with A $\beta$ immunostaining (Fig. 3D, arrowheads).

\section{Time-Activity Data of ${ }^{11} \mathrm{C}-\mathrm{BF}-227$ in Clinical PET Study}

No toxic event was observed in the current clinical trial of ${ }^{11} \mathrm{C}-\mathrm{BF}-227$. The SUV time-activity curves from ${ }^{11} \mathrm{C}$ BF-227 PET in AD patients and all normal subjects are shown in Figure 4. Both groups showed rapid entry of ${ }^{11} \mathrm{C}$ BF-227 into gray matter areas. In AD patients, the frontal, temporal, and parietal cortices, areas known to contain high concentrations of fibrillar amyloid plaques in $\mathrm{AD}$, retained ${ }^{11} \mathrm{C}-\mathrm{BF}-227$ to a greater extent during the later time points compared with normal subjects (Figs. 4A-4C). When the 2 groups were compared, a significant difference in timeactivity curves was observed in the frontal (Fig. 4A), lateral temporal (Fig. 4B), parietal (Fig. 4C), and visual association cortices (data not shown). In contrast, time-activity curves in the cerebellum (Fig. 4D), an area lacking fibrillar amyloid plaques, were nearly identical in normal subjects and AD patients. The subcortical white matter region showed relatively lower entry and slower clearance than gray matter areas but no significant difference in time-activity curves between the 2 groups (data not shown). In the comparison of time-activity curves in the cortical areas and cerebellum, $\mathrm{AD}$ patients showed a significant difference in time-activity curves over 10 min after administration of ${ }^{11} \mathrm{C}-\mathrm{BF}-227$, but normal subjects showed no significant differences.

SUV Images in AD Patients and Normal Control Subjects

SUV images summed over 20-40 min after injection of an aged normal subject (70-y-old woman) and an AD patient (68-y-old woman; MMSE score $=21)$ are shown in Figure 5. Cortical retention of ${ }^{11} \mathrm{C}-\mathrm{BF}-227$, especially in the basal portion of the frontal, temporal, and parietal region, was evident in the $\mathrm{AD}$ patient, in contrast with the images of the aged normal subject. This pattern of distribution is consistent with the findings of neuritic plaque distribution in postmortem AD brains (18). Higher retention of ${ }^{11} \mathrm{C}-\mathrm{BF}-227$ was also observed in the brainstem and thalamus; however, similar retention in these areas was detected in the aged normal subject. ${ }^{11} \mathrm{C}-\mathrm{BF}-227$ uptake in the cerebellum was relatively sparse in both the aged normal subject and the AD patient.

\section{Comparisons of Regional SUVs and SUV Ratios}

In the quantitative comparison of regional SUVs between 40 and 60 min after administration, cortical regions showed the tendency to be increased in AD patients; however, the difference was not significant because of the large individual difference in SUVs. SUVs in the thalamus, pons, and white matter were similar in the 3 groups. Because there were no plaques in the cerebellum, there was no BF-227 binding and no significant difference in the SUV between $\mathrm{AD}$ and normal groups, indicating that the cerebellum is adequate as a reference region. Therefore, the ratio of regional SUV to cerebellar SUV (SUV ratio) was calculated as an index of ${ }^{11} \mathrm{C}-\mathrm{BF}-227$ retention. This analysis successfully reduced the intersubject variability, as reflected in low SD values (Table 2). The mean SUV ratio for the frontal, lateral temporal, parietal, temporooccipital, occipital, anterior and posterior cingulate cortices, and striatum was significantly greater in AD patients than that in aged normal subjects (Table 2; Fig. 6). Notably, the SUV ratio in the lateral temporal cortex showed no overlap between AD patients and normal control subjects (Fig. 6). The SUV ratio in the medial temporal cortex, thalamus,
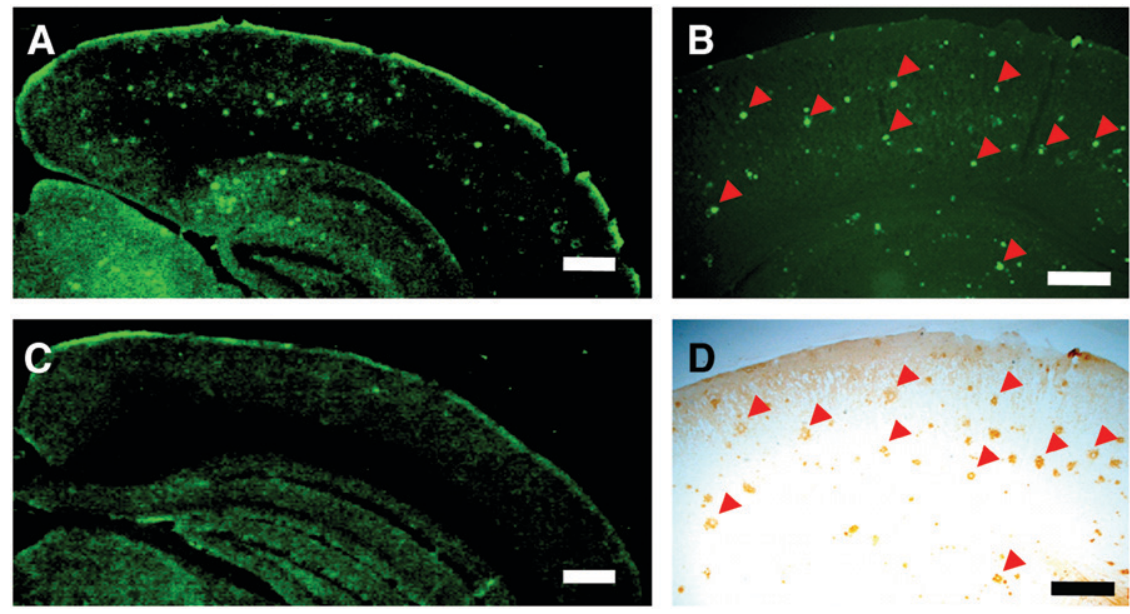

FIGURE 3. In vivo binding of BF-227 to amyloid plaques in PS1/APP transgenic mouse. In brain sections from PS1/APP transgenic mouse after intravenous in-

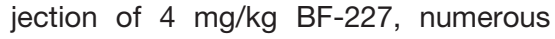
fluorescent spots were observed in neocortex and hippocampus of brain (A and B). In contrast, no fluorescent spots were observed in brain of wild-type mouse (C). Distribution of plaques labeled with BF227 corresponded well with $A \beta$ immunostaining in same section ( $B$ and $D$, arrowheads). 
FIGURE 4. Time-activity data for ${ }^{11} \mathrm{C}-$ BF-227 PET in humans. SUV timeactivity curves of ${ }^{11} \mathrm{C}-\mathrm{BF}-227$ in frontal cortex $(A)$, temporal cortex $(B)$, parietal cortex (C), and cerebellum (D) are shown. Each point represents mean \pm SEM of data from 7 AD patients and 7 normal control subjects.

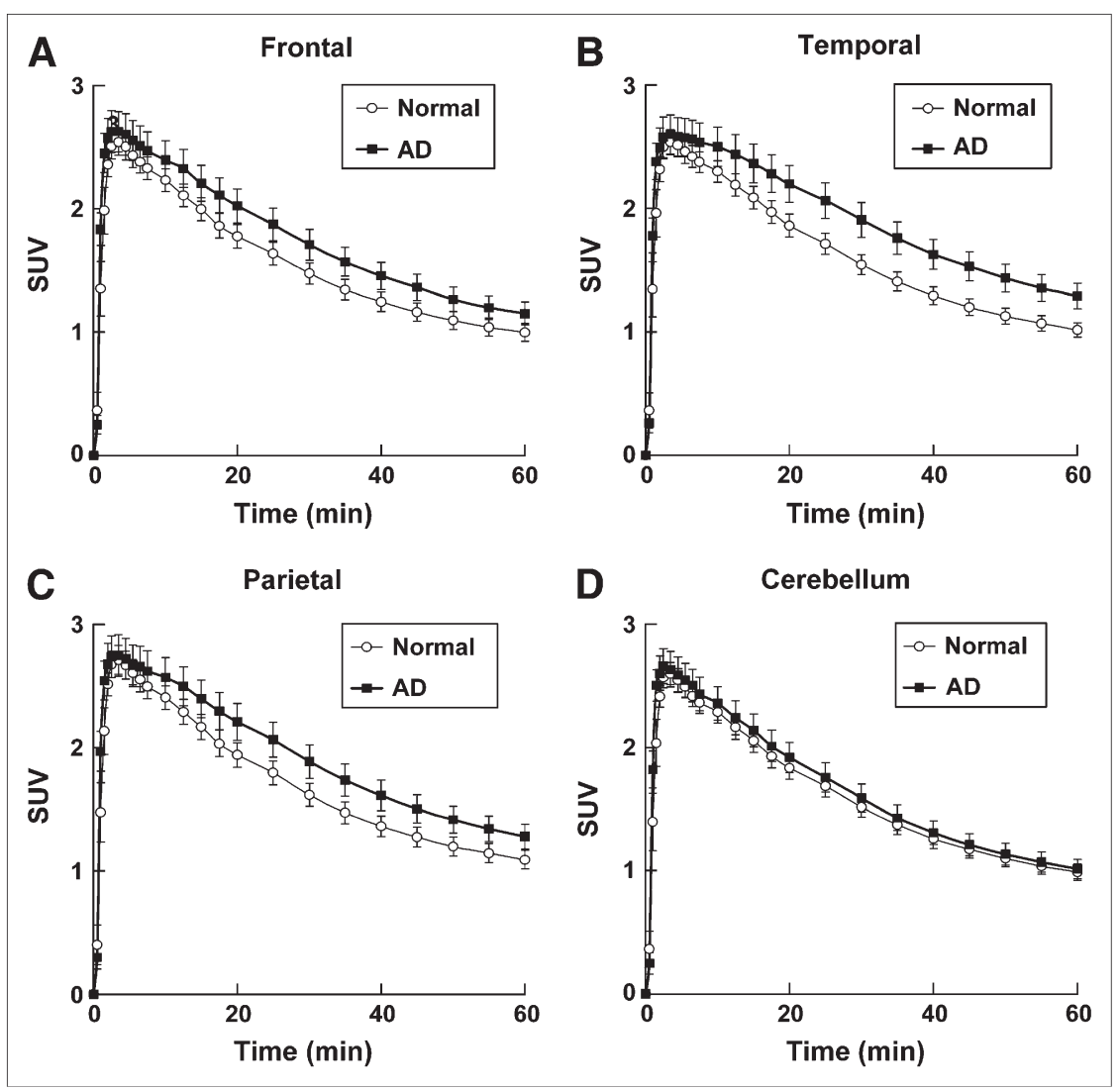

pons, and white matter was nearly identical in AD patients and normal subjects. The effect size between AD patients and aged normal subjects was highest in the lateral temporal cortex, which was followed by the parietal, anterior cingulate, and frontal cortices, and was lowest in the medial temporal, thalamus, and pons (Table 2). No significant difference was observed in any brain regions between young normal and aged normal subjects, although aged individuals tended to exhibit a higher SUV ratio in the frontal cortex than young individuals (data not shown).

\section{Voxel-by-Voxel Analysis of ${ }^{11} \mathrm{C}-\mathrm{BF}-227$ PET Images}

In comparison with aged normal subjects, $\mathrm{AD}$ patients showed significantly higher uptake of ${ }^{11} \mathrm{C}-\mathrm{BF}-227$ in the bilateral temporoparietal region $([50,-56,6], Z=5.41$, $k=22,823)$, including the posterior cingulate cortex and the left middle frontal gyrus ([-26, 24, 40], $Z=3.79, k=$ 1,401) in SPM analysis (Fig. 7). These areas corresponded well with the region containing a high density of neuritic plaques. In contrast, no significant region was detected showing lower uptake of ${ }^{11} \mathrm{C}-\mathrm{BF}-227$ in the $\mathrm{AD}$ group than that in the normal group.

\section{DISCUSSION}

BF-227 was designed to improve BBB penetration and clearance from normal brain tissue, without deteriorating the high binding affinity of benzoxazole derivatives to $A \beta$.
Several lipophilic compounds have been reported as potential amyloid imaging probes. 2-(1-\{6-[(2-Fluoroethyl)(methyl) amino]-2-naphthyl \}ethylidene)malononitrile (FDDNP) was introduced as the first BBB-permeable compound for in

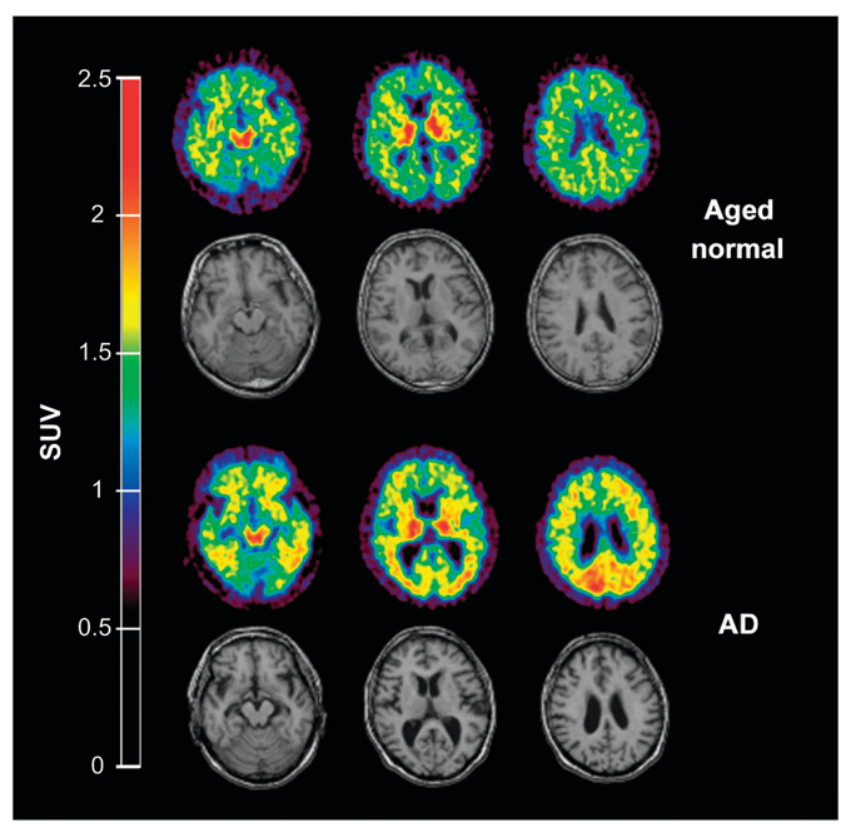

FIGURE 5. Mean SUV images between 20 and 40 min after injection of ${ }^{11} \mathrm{C}-\mathrm{BF}-227$ in aged normal subject (top, 70-y-old woman) and $A D$ patient (bottom, 68-y-old woman). Coregistered MR images are shown below PET images. 
TABLE 2

Regional SUV and Regional-to-Cerebellar SUV Ratio of ${ }^{11} \mathrm{C}-\mathrm{BF}-227$ in Normal Subjects and AD Patients

\begin{tabular}{|c|c|c|c|c|c|c|c|}
\hline \multirow[b]{2}{*}{ Distribution } & \multicolumn{3}{|c|}{ SUV } & \multicolumn{3}{|c|}{ SUV ratio } & \multirow{2}{*}{$\begin{array}{c}\text { Cohen's } d, \\
\text { Aged normal vs. AD }\end{array}$} \\
\hline & All normal & Aged normal & $A D$ & All normal & Aged normal & $A D$ & \\
\hline Frontal & $1.13 \pm 0.23$ & $1.11 \pm 0.24$ & $1.24 \pm 0.27$ & $0.99 \pm 0.04$ & $0.99 \pm 0.05$ & $1.13 \pm 0.06^{\star}$ & 2.54 \\
\hline Lateral temporal & $1.16 \pm 0.22$ & $1.15 \pm 0.23$ & $1.38 \pm 0.30$ & $1.03 \pm 0.05$ & $1.02 \pm 0.04$ & $1.25 \pm 0.06^{*}$ & 4.51 \\
\hline Parietal & $1.22 \pm 0.24$ & $1.19 \pm 0.24$ & $1.36 \pm 0.30$ & $1.08 \pm 0.06$ & $1.06 \pm 0.05$ & $1.24 \pm 0.06^{\star}$ & 3.26 \\
\hline Temporooccipital & $1.22 \pm 0.23$ & $1.21 \pm 0.24$ & $1.35 \pm 0.27$ & $1.08 \pm 0.06$ & $1.08 \pm 0.06$ & $1.23 \pm 0.09^{\star}$ & 1.96 \\
\hline Occipital & $1.23 \pm 0.23$ & $1.21 \pm 0.24$ & $1.32 \pm 0.26$ & $1.09 \pm 0.06$ & $1.08 \pm 0.06$ & $1.20 \pm 0.07^{*}$ & 1.84 \\
\hline Anterior cingulate & $1.19 \pm 0.26$ & $1.16 \pm 0.26$ & $1.27 \pm 0.26$ & $1.04 \pm 0.04$ & $1.03 \pm 0.04$ & $1.16 \pm 0.06^{*}$ & 2.55 \\
\hline Posterior cingulate & $1.28 \pm 0.25$ & $1.24 \pm 0.25$ & $1.38 \pm 0.26$ & $1.13 \pm 0.08$ & $1.11 \pm 0.08$ & $1.26 \pm 0.04^{*}$ & 2.37 \\
\hline Medial temporal & $1.33 \pm 0.24$ & $1.31 \pm 0.25$ & $1.31 \pm 0.27$ & $1.18 \pm 0.07$ & $1.17 \pm 0.07$ & $1.20 \pm 0.10$ & 0.35 \\
\hline Striatum & $1.57 \pm 0.34$ & $1.52 \pm 0.34$ & $1.62 \pm 0.34$ & $1.38 \pm 0.08$ & $1.35 \pm 0.06$ & $1.47 \pm 0.06^{\star}$ & 2.00 \\
\hline Thalamus & $1.78 \pm 0.44$ & $1.70 \pm 0.41$ & $1.73 \pm 0.36$ & $1.56 \pm 0.12$ & $1.51 \pm 0.09$ & $1.58 \pm 0.11$ & 0.70 \\
\hline Pons & $1.90 \pm 0.34$ & $1.87 \pm 0.34$ & $1.91 \pm 0.39$ & $1.68 \pm 0.08$ & $1.67 \pm 0.08$ & $1.74 \pm 0.09$ & 0.82 \\
\hline White matter & $1.64 \pm 0.27$ & $1.61 \pm 0.28$ & $1.69 \pm 0.33$ & $1.45 \pm 0.11$ & $1.44 \pm 0.11$ & $1.55 \pm 0.12$ & 0.96 \\
\hline Cerebellum & $1.14 \pm 0.23$ & $1.13 \pm 0.24$ & $1.10 \pm 0.23$ & & & & \\
\hline
\end{tabular}

vivo imaging of amyloid. FDDNP specifically binds to both SPs and NFTs in AD brain sections (19). After intravenous injection of FDDNP, greater accumulation was observed in SP- and NFT-rich areas of the human brain (20). Thioflavin- $T$ derivatives without any positive charge also show high permeability of the BBB. One of these compounds, PIB, was applied in a human PET study and enabled successful differentiation between AD patients and healthy normal individuals (5). Another amyloid-imaging agent, SB-13, was also applied in a human PET study and exhibited binding properties similar to those of PIB (21). Several iodinated agents, 6-iodo-2-(4'-dimethylamino-) phenyl-imidazo[1,2-a]-pyridine (IMPY) and I-stilbene, have also been explored for use as SPECT probes (22). Although validation remains necessary to determine whether retention of these agents in the neocortex truly reflects the level of amyloid deposition, such findings suggest the potential usefulness of this technique for early diagnosis of AD.

The results of the in vitro binding experiment indicate that binding of BF-227 reflects the amount of $A \beta$ fibril deposition. In neuropathologic staining of AD brain sections, the fluorescence intensity of BF-227 is highest in the core region of mature amyloid deposits, which contain dense fibrils of $A \beta$. Conversely, diffuse plaques containing fewer A $\beta$ fibrils are faintly stained by BF-227. SPs in the cerebellum are predominantly of the nonfibrillar type $(23,24)$, and BF-227 only faintly stained diffuse amyloid plaques in the cerebellum. Thus, the absence of ${ }^{11} \mathrm{C}-\mathrm{BF}-227$ accumulation in the cerebellum of $\mathrm{AD}$ patients suggests the binding preference of this compound for fibrillar $A \beta$. This finding also indicates that the cerebellum is suitable as a reference region in the quantitative analysis of ${ }^{11} \mathrm{C}-\mathrm{BF}-227$ PET data.

PIB is currently the most successful of several amyloidimaging agents. A clinical PET study in AD patients showed higher uptake of PIB in cortical areas and striatum, particularly the frontal and parietal cortices (5-7). In contrast, the current study demonstrated higher cortical retention of ${ }^{11} \mathrm{C}$ BF-227 in the temporoparietal-occipital region rather than that in the frontal cortex and the striatum in AD patients. Both agents are considered to preferentially bind to the $\beta$-sheet structure of $\mathrm{A} \beta$ fibrils. What other factors could

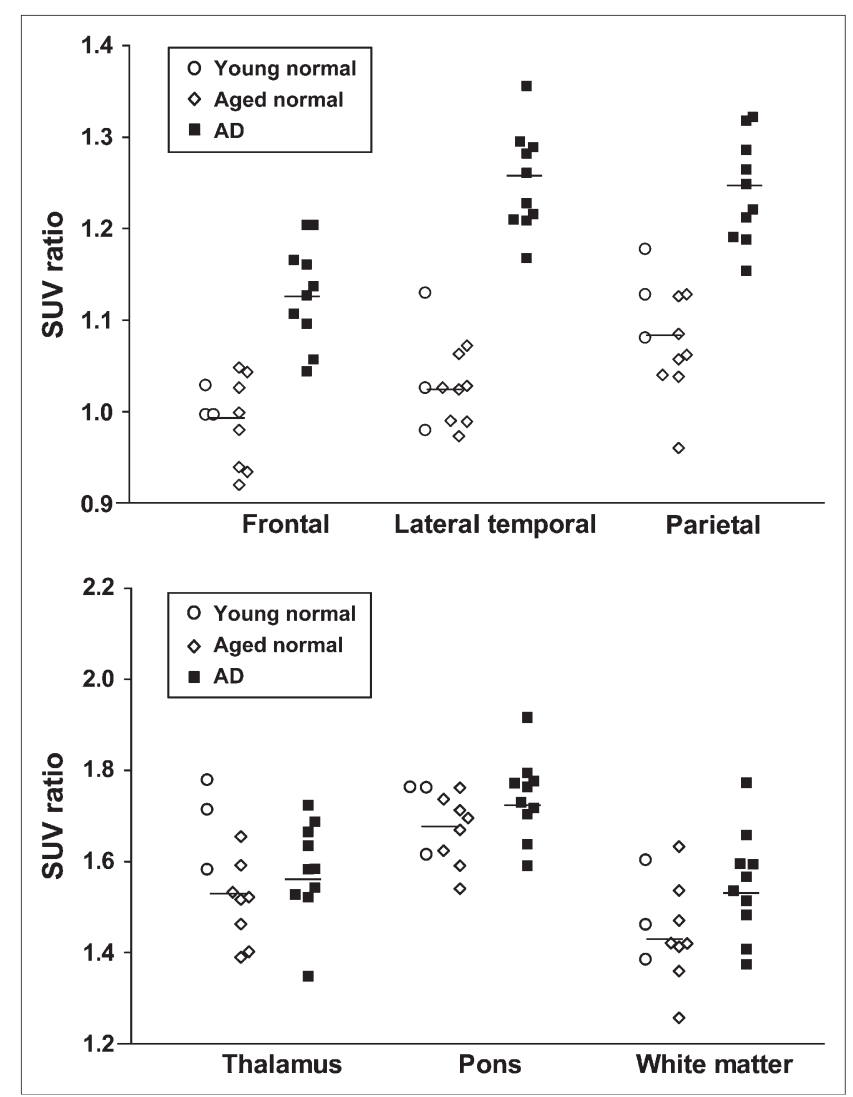

FIGURE 6. ROI/cerebellar SUV ratio in young normal subjects $(\bigcirc)$, aged normal subjects $(\diamond)$, and AD patients $(\square)$. Vertical bar represents average SUV ratio in all normal subjects $(n=11)$ and AD patients $(n=10)$. 


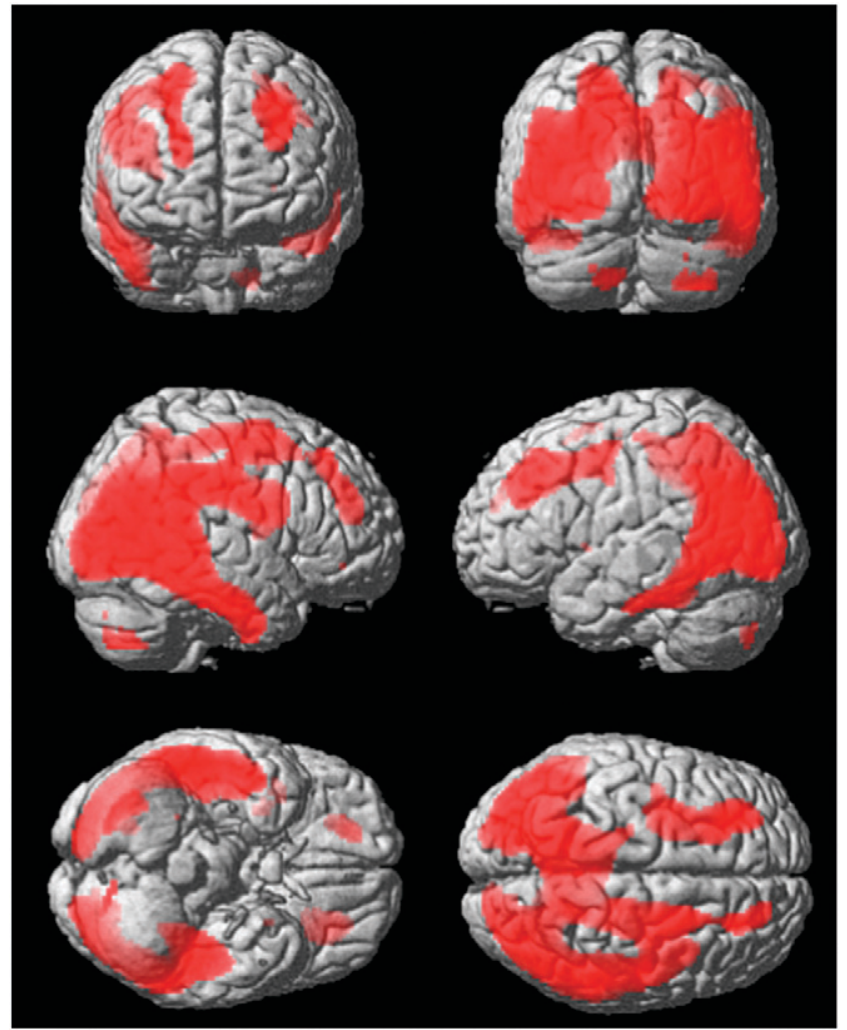

FIGURE 7. Brain regions show significantly elevated SUVs in $A D$ patients compared with data from aged healthy subjects ( $P<0.001$, uncorrected for multiple comparisons).

have caused the difference of tracer distribution between previous PIB studies and the current BF-227 study? Generally, substantial individual variations exist in the amount and spatial distribution of amyloid deposition in AD. Thus, the discrepancy might be partially attributable to a difference in sample populations between studies. To settle this issue, a direct comparison study between PIB and BF-227 should be conducted using the same sample populations. SP is a heterogeneous class of protein aggregates with a $\beta$-pleated structure. Compact plaques consist of a dense central core of amyloid fibrils, and noncompact plaques contain less fibrillar $A \beta$ (25). Therefore, it would be expected that the lower-affinity compound would tend to detect only SPs with dense $A \beta$ fibrils and that the higher-affinity compound could bind SPs with both dense and moderately fibrillar $\mathrm{A} \beta$. In $\mathrm{AD}$ patients, the difference between cortical and cerebellar SUV in ${ }^{11} \mathrm{C}-\mathrm{BF}-227$ PET was less than that in PIB PET (5-7), suggesting that the in vivo binding affinity of BF-227 to $A \beta$ deposits is relatively lower than that of PIB. If so, BF-227 binds more preferentially to dense amyloid deposits than PIB. Previous neuropathologic studies have indicated that neuritic plaque densities are highest in the neocortex, especially the temporoparietooccipital region, and lowest in the cerebellum $(18,26)$. Data from SPM analysis are consistent with the postmortem distribution of neuritic plaque deposition in $\mathrm{AD}$ patients. Therefore, the difference in cortical distribution between
BF-227 and PIB might be due to the difference in binding affinity to $A \beta$ fibrils. A PET probe binding selectively to neuritic plaques would be less subject to $A \beta$ pathology in the normal aging process. Thus, use of ${ }^{11} \mathrm{C}-\mathrm{BF}-227$ PET will allow accurate diagnosis of $\mathrm{AD}$ and might reduce false-positive findings in normal individuals. ${ }^{11} \mathrm{C}-\mathrm{BF}-227$ PET might also be useful for tracking the progression of fibrillar $\mathrm{A} \beta$ deposition in $\mathrm{AD}$ patients. Longitudinal PET investigation of $\mathrm{AD}$ patients will elucidate the utility of this imaging technique for monitoring disease progression in AD.

NFTs stained faintly with BF-227, suggesting that BF227 has a relatively lower binding affinity to NFTs than SPs, which might explain the lack of significant difference in the medial temporal SUV of ${ }^{11} \mathrm{C}-\mathrm{BF}-227$. However, $3 \mathrm{AD}$ patients (AD 1, AD 2, and AD 3 in Table 1) exhibiting high BF-227 accumulation in the cerebral cortex showed higher accumulation in the medial temporal cortex than the other AD patients. This finding might reflect the increasing deposition of amyloid plaques in the medial temporal cortex of the $3 \mathrm{AD}$ patients. Thalamic and white matter accumulation of ${ }^{11} \mathrm{C}-\mathrm{BF}-227$ was considerable in both $\mathrm{AD}$ patients and normal subjects. Retention levels of ${ }^{11} \mathrm{C}-\mathrm{BF}-227$ in these regions were nearly identical between normal and $\mathrm{AD}$ groups. Therefore, these retentions are not likely to reflect AD-specific pathology. BF-227 retention in these sites may be related to the many myelinated fibers present in these structures, because myelin basic protein-one of the major myelin proteins in the brain-partially shares the same structure with amyloid fibrils, and some $\beta$-sheet binding agents bind to this protein (27-29). Clearance of ${ }^{11} \mathrm{C}-\mathrm{BF}-$ 227 from normal brain tissue was slower than that of PIB. This might be caused by the difference in lipophilicity between BF-227 and PIB. BF-227 $(\log P=1.75)$ is more lipophilic than PIB ( $\log P=1.20$ ) (30) because, unlike PIB, BF-227 does not have a hydroxy group. Compounds that are too lipophilic will be bound by plasma protein and undergo rapid metabolism by the liver; therefore, they may display reduced brain uptake. Moreover, lipophilic radioligands display a higher nonspecific binding in the brain and, thus, high nonspecific binding may explain the moderate difference in BF-227 uptake between AD patients and normal control subjects. In general, the introduction of a hydroxy group into a molecule changes the partition coefficient toward more hydrophilicity. Therefore, the hydroxylated BF-227 derivative would be expected to show faster clearance from normal brain tissue and a better signalto-noise ratio than BF-227. We are now implementing the optimizing process to reduce white matter retention and plan to apply the optimized compound to the candidate for an ${ }^{18}$ F-labeled PET probe.

\section{CONCLUSION}

The present study demonstrated that the benzoxazole derivative BF-227 displays high binding affinity to amyloid 
plaques and high BBB permeability. The current clinical trial indicated that BF-227 has adequate safety to be used clinically as a PET probe. ${ }^{11} \mathrm{C}-\mathrm{BF}-227$ PET demonstrated significant retention of this agent in sites with a preference for the deposition of dense amyloid plaques and distinctly differentiated between $\mathrm{AD}$ patients and normal individuals. Collectively, these findings suggest that ${ }^{11} \mathrm{C}-\mathrm{BF}-227$ is useful for early diagnosis of AD.

\section{ACKNOWLEDGMENTS}

This study was supported by the Program for the Promotion of Fundamental Studies in Health Science by the National Institute of Biomedical Innovation, the Special Coordination Funds for Promoting Science and Technology, the Industrial Technology Research Grant Program in 2004 from the New Energy and Industrial Technology Development Organization of Japan, Health and Labour Sciences Research Grants for Translational Research from the Ministry of Health, Astrazeneca Research Grant 2004, and the Novartis Foundation for Gerontological Research. We appreciate the technical assistance of Dr. Shoichi Watanuki and Dr. Yoichi Ishikawa in the clinical PET studies and Dr. Motohisa Kato in the imaging analysis. We also thank Dr. Hiroyasu Akatsu and Dr. Takayuki Yamamoto for supplying brain samples.

\section{REFERENCES}

1. Morris JH, Nagy Z. Alzheimer's disease. In: Esiri MM, Lee VM, Trojanowski JQ, eds. The Neuropathology of Dementia. 2nd ed. Cambridge, U.K.: Cambridge University Press; 2004:161-206.

2. Goldman WP, Price JL, Storandt M, et al. Absence of cognitive impairment or decline in preclinical Alzheimer's disease. Neurology. 2001;56:361-367.

3. Price JL, Morris JC. Tangles and plaques in nondemented aging and "preclinical" Alzheimer's disease. Ann Neurol. 1999;45:358-368.

4. Aisen PS. The development of anti-amyloid therapy for Alzheimer's disease: from secretase modulators to polymerisation inhibitors. CNS Drugs. 2005;19:989-996.

5. Klunk WE, Engler H, Nordberg A, et al. Imaging brain amyloid in Alzheimer's disease with Pittsburgh Compound-B. Ann Neurol. 2004;55:306-319.

6. Price JC, Klunk WE, Lopresti BJ, et al. Kinetic modeling of amyloid binding in humans using PET imaging and Pittsburgh Compound-B. J Cereb Blood Flow Metab. 2005;25:1528-1547.

7. Lopresti BJ, Klunk WE, Mathis CA, et al. Simplified quantification of Pittsburgh Compound B amyloid imaging PET studies: a comparative analysis. J Nucl Med. 2005;46:1959-1972.

8. Price JL. Diagnostic criteria for Alzheimer's disease. Neurobiol Aging. 1997; 18(suppl):S67-S70.

9. Wang J, Dickson DW, Trojanowski JQ, Lee VM. The levels of soluble versus insoluble brain $\mathrm{A} \beta$ distinguish Alzheimer's disease from normal and pathologic aging. Exp Neurol. 1999;158:328-337.
10. Okamura N, Suemoto T, Shimadzu H, et al. Styrylbenzoxazole derivatives for in vivo imaging of amyloid plaques in the brain. J Neurosci. 2004;24:25352541.

11. Okamura N, Suemoto T, Shiomitsu T, et al. A novel imaging probe for in vivo detection of neuritic and diffuse amyloid plaques in the brain. J Mol Neurosci. 2004;24:247-255.

12. Shimadzu H, Suemoto T, Suzuki M, et al. Novel probes for imaging amyloid- $\beta$ : F-18 and C-11 labeling of 2-(4-aminostyryl)benzoxazole derivatives. J Labelled Compds Radiopharm. 2004;47:181-190.

13. Jewett DM. A simple synthesis of $\left[{ }^{11} \mathrm{C}\right]$ methyl triflate. Appl Radiat Isot. 1992;43:1383-1385.

14. Iwata R, Pascali C, Bogni A, Miyake Y, Yanai K, Ido T. A simple loop method for the automated preparation of $\left[{ }^{11} \mathrm{C}\right]$ raclopride from $\left[{ }^{11} \mathrm{C}\right]$ methyl triflate. Appl Radiat Isot. 2001;55:17-22.

15. Holcomb L, Gordon MN, McGowan E, et al. Accelerated Alzheimer-type phenotype in transgenic mice carrying both mutant amyloid precursor protein and presenilin 1 transgenes. Nat Med. 1998;4:97-100.

16. Friston KJ, Holmes AP, Worsley KJ, Poline JP, Frith CD, Frackowiack RSJ. Statistical parametric maps in functional imaging: a general linear approach. Hum Brain Mapp. 1995;2:189-210.

17. Higuchi M, Tashiro M, Arai H, et al. Glucose hypometabolism and neuropathological correlates in brains of dementia with Lewy bodies. Exp Neurol. 2000; 162:247-256.

18. Arnold SE, Hyman BT, Flory J, Damasio AR, Van Hoesen GW. The topographical and neuroanatomical distribution of neurofibrillary tangles and neuritic plaques in the cerebral cortex of patients with Alzheimer's disease. Cereb Cortex. 1991;1:103-116.

19. Agdeppa ED, Kepe V, Liu J, et al. Binding characteristics of radiofluorinated 6-dialkylamino-2-naphthylethylidene derivatives as positron emission tomography imaging probes for beta-amyloid plaques in Alzheimer's disease. J Neurosci. 2001;21:RC189:1-5.

20. Shoghi-Jadid K, Small GW, Agdeppa ED, et al. Localization of neurofibrillary tangles and beta-amyloid plaques in the brains of living patients with Alzheimer disease. Am J Geriatr Psychiatry. 2002;10:24-35.

21. Verhoeff NP, Wilson AA, Takeshita S, et al. In-vivo imaging of Alzheimer disease beta-amyloid with $\left[{ }^{11} \mathrm{C}\right] \mathrm{SB}-13$ PET. Am J Geriatr Psychiatry. 2004;12: 584-595.

22. Kung HF, Kung MP, Zhuang ZP, et al. Iodinated tracers for imaging amyloid plaques in the brain. Mol Imaging Biol. 2003;5:418-426.

23. Yamaguchi H, Hirai S, Morimatsu M, Shoji M, Nakazato Y. Diffuse type of senile plaques in the cerebellum of Alzheimer-type dementia demonstrated by beta protein immunostain. Acta Neuropathol (Berl). 1989;77:314-319.

24. Yamazaki T, Yamaguchi H, Nakazato Y, Ishiguro K, Kawarabayashi T, Hirai S. Ultrastructural characterization of cerebellar diffuse plaques in Alzheimer's disease. J Neuropathol Exp Neurol. 1992;51:281-286.

25. Dickson DW. The pathogenesis of senile plaques. J Neuropathol Exp Neurol. 1997;56:321-339.

26. Joachim CL, Morris JH, Selkoe DJ. Diffuse senile plaques occur commonly in the cerebellum in Alzheimer's disease. Am J Pathol. 1989;135:309-319.

27. Bjelke B, Seiger A. Morphological distribution of MBP-like immunoreactivity in the brain during development. Int J Dev Neurosci. 1989;7:145-164.

28. Ridsdale RA, Beniac DR, Tompkins TA, Moscarello MA, Harauz G. Threedimensional structure of myelin basic protein. II. Molecular modeling and considerations of predicted structures in multiple sclerosis. J Biol Chem. 1997; 272:4269-4275.

29. Stankoff B, Wang Y, Bottlaender M, et al. Imaging of CNS myelin by positronemission tomography. Proc Natl Acad Sci U S A. 2006;103:9304-9309.

30. Mathis CA, Wang Y, Holt DP, Huang GF, Debnath ML, Klunk WE. Synthesis and evaluation of ${ }^{11} \mathrm{C}$-labeled 6-substituted 2-arylbenzothiazoles as amyloid imaging agents. J Med Chem. 2003;46:2740-2754. 